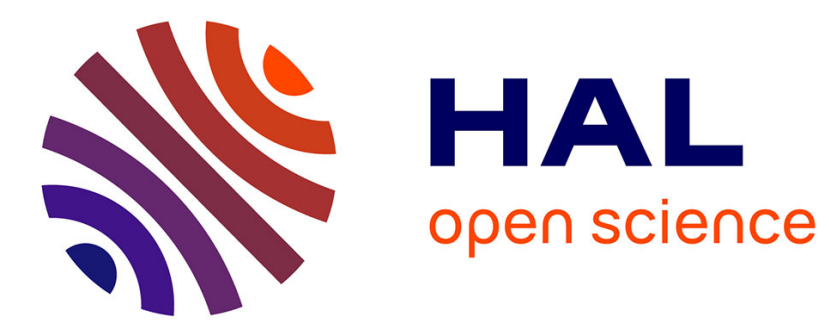

\title{
Portées et limites du lot virtuel : une étude exploratoire
} Ouidade Sabri-Zaaraoui, Béatrice Parguel, Pauline de Pechpeyrou

\section{To cite this version:}

Ouidade Sabri-Zaaraoui, Béatrice Parguel, Pauline de Pechpeyrou. Portées et limites du lot virtuel : une étude exploratoire. Décisions Marketing, 2008, 50, pp.49-57. halshs-00271745

\section{HAL Id: halshs-00271745 https://shs.hal.science/halshs-00271745}

Submitted on 12 Jan 2012

HAL is a multi-disciplinary open access archive for the deposit and dissemination of scientific research documents, whether they are published or not. The documents may come from teaching and research institutions in France or abroad, or from public or private research centers.
L'archive ouverte pluridisciplinaire HAL, est destinée au dépôt et à la diffusion de documents scientifiques de niveau recherche, publiés ou non, émanant des établissements d'enseignement et de recherche français ou étrangers, des laboratoires publics ou privés. 


\title{
PORTEES ET LIMITES DU LOT VIRTUEL : UNE ETUDE EXPLORATOIRE
}

\author{
Sabri-Zaaraoui O. ${ }^{1}$, Parguel B. ${ }^{2}$ et De Pechpeyrou P. ${ }^{3}$
}

${ }^{1}$ Ouidade Sabri-Zaaraoui, A.T.E.R., DRM-DMSP Université Paris-Dauphine, 3, rue Saint Exupéry 92360 Meudon-La-Forêt, ouidade.sabri@ yahoo.fr, Tél 06.63.09.23.69

2 Béatrice Parguel, A.T.E.R., DRM-DMSP Université Paris-Dauphine, 3, avenue du Président Franklin Roosevelt 92330 Sceaux, beatrice.parguel@wanadoo.fr, Tél 01.47.02.06.25

${ }^{3}$ Pauline De Pechpeyrou, A.T.E.R., Lille II (ESA), ESSEC et DRM-DMSP Université Paris-Dauphine, 20, rue Jean Nicot 75007 Paris, depechpeyrou@essec.fr, Tél 01.45.55.10.18

Les auteurs souhaitent remercier L. Battais (Directeur Général de la société MarketingScan) et P. Denis (Directeur Général de la société Le Site Marketing) pour leur avoir fourni les données nécessaires, ainsi que les lecteurs anonymes pour leurs commentaires constructifs. 


\title{
PORTEES ET LIMITES DU LOT VIRTUEL : UNE ETUDE EXPLORATOIRE
}

\section{Résumé :}

Le coût élevé de mise en œuvre des techniques promotionnelles traditionnelles amène les distributeurs et les industriels à rechercher de nouveaux formats de promotion, parmi lesquels le lot dit «virtuel ». Après avoir présenté cette technique et ses enjeux, l'article analyse les principaux avantages qu'en retirent les acteurs de la distribution, ainsi que ses contraintes. Il s'interroge dans un second temps sur les réactions des consommateurs face au lot virtuel à l'aide d'une approche qualitative exploratoire. Les facteurs clés de succès de l'implantation du lot virtuel en magasin sont proposés à la suite de ce double éclairage managers et consommateurs.

Mots clés : Lot virtuel - Coûts et bénéfices - Relations industriels-distributeurs.

\section{BENEFITS AND LIMITS OF VIRTUAL BUNDLING: AN EXPLORATORY STUDY}

\begin{abstract}
:
The high costs associated with traditional promotional techniques lead retailers and industrials to adopt new promotional formats, especially "separate-item bundles". After presenting this promotional format and its managerial stakes, the article first analyzes the advantages and constraints that separate-item bundles present for actors in the retail industry. We next use an exploratory qualitative approach to shed light on consumers' reactions to such a new promotional format. This double focus - on managers and consumers - leads us to propose key success factors for separate-item bundles implementation at the point of purchase.
\end{abstract}

Key words: Separate-item bundles - Costs and benefits - Industrial-retailer relations. 


\section{Introduction}

Les promotions sont de plus en plus utilisées par les distributeurs pour attirer les clients dans les magasins et augmenter leur chiffre d'affaires. Parmi les techniques promotionnelles les plus utilisées pour développer les ventes figurent les lots classiques (ou lots «réels ») et les formats spécifiques, qui comptent également parmi les plus coûteuses. Distributeurs et industriels peuvent toutefois désormais recourir au lot virtuel pour éviter une partie de ces coûts.

Comme le lot réel, le lot virtuel conditionne l'obtention d'une gratuité ou d'une réduction à l'achat simultané d'un nombre déterminé d'unités de produits $(8,13)$. Néanmoins, si les unités de produits sont physiquement assemblées dans une même unité de vente (sur-emballage) dans le cas du lot réel, elles sont présentées séparément dans le cas du lot virtuel (5). Le client constitue alors lui-même son lot dans son panier et la vérification des conditions d'octroi de l'avantage est effectuée lors du passage en caisse. Cette caractéristique explique l'usage alternatif de la terminologie «lot électronique » par certains acteurs pour désigner le lot virtuel. Les principales variantes du lot virtuel sont présentées dans l'encadré 1.

[insérer encadré 1]

Le lot virtuel connaît actuellement un développement rapide dans la grande distribution en France (encadré 2). Toutefois, peu de travaux ont été consacrés à l'explication d'un tel essor comme à l'étude de son efficacité du point de vue du consommateur. Ces observations conduisent donc à une double interrogation managériale : quelles sont les forces et les faiblesses du lot virtuel par rapport au lot réel pour les acteurs de la grande consommation? comment le consommateur perçoit-il le lot virtuel et comment, en conséquence, optimiser sa mise en œuvre? Pour répondre à ces questions, nous exposerons tout d'abord l'enjeu managérial que représente la technique promotionnelle du lot virtuel, avant d'aborder les conclusions tirées des deux études qualitatives menées auprès d'experts et de consommateurs.

[insérer encadré 2] 


\section{Le lot virtuel, un enjeu managérial important}

Le lot appartient aux techniques promotionnelles de prix. Il fait donc appel aux motivations économiques de l'acheteur pour le conduire à acheter immédiatement le produit, et éventuellement à le stocker. L'objectif pour les industriels comme pour les distributeurs est ainsi de développer les volumes vendus et par conséquent le chiffre d'affaires réalisé. La mise en œuvre de cette technique promotionnelle engendre cependant tant des frais variables, liés en particulier au succès remporté par l'opération promotionnelle (coût du sur-emballage ou d'une erreur de prévision des ventes par magasin), que des frais fixes (création d'un code barre d'identification EAN et d'une communication spécifique, coût de l'espace utilisé). L'importance de ces coûts au regard des objectifs escomptés (6) conduit les partenaires de la chaîne commerciale à rechercher de nouvelles formes de collaboration dans la définition des packagings, la gestion de l'assortiment et des approvisionnements ou l'organisation d'opérations de trade marketing (4).

Le lot virtuel en est une illustration : il vise à répondre aux préoccupations des acteurs de la grande distribution, en jugulant les coûts inhérents aux lots réels, tout en préservant, voire en développant, les bénéfices qui s'y trouvent associés.

Le lot virtuel répond d'abord à l'objectif d'augmentation des volumes vendus traditionnellement assigné à la technique du lot. En effet, il procure potentiellement le même avantage monétaire que le lot réel, que ce soit sous la forme de produit gratuit ou d'une réduction monétaire. En allant plus loin, il se pourrait même que le consommateur associe au lot virtuel un bénéfice économique supérieur à celui du lot réel. En effet, de nombreux travaux suggèrent que l'annonce d'un prix de référence conduit à une meilleure évaluation de l'offre (2), et le lot virtuel affiche justement un prix de référence - le prix d'achat unitaire - en sus du prix du lot, ce que ne fait pas le lot réel. De plus, lorsqu'il se présente sous une forme hétérogène (variété ou gamme), le lot virtuel atteint également un objectif de pénétration, caractéristique des techniques promotionnelles d'essai. Il offre alors au consommateur l'opportunité de découvrir une autre variété ou d'essayer un nouveau produit. 
Le lot virtuel est ensuite l'occasion de renforcer la relation entre l'industriel et le distributeur. Au-delà d'une réduction des coûts promotionnels pour les acteurs, le lot virtuel correspond pour les enseignes à une nouvelle stratégie de communication susceptible de véhiculer une image prix plus novatrice. Le lot virtuel permet également au point de vente de varier l'offre à l'infini et de la changer à loisir, plus fréquemment que pour la promotion classique, qui se trouve contrainte par ses paquets identifiés et ses volumes stockés. Ce dernier point apparaît désormais comme une attente primordiale chez les directeurs de magasin, qui souhaitent que leurs fournisseurs adaptent davantage au magasin les actions promotionnelles négociées en centrale (9). Le lot virtuel s'inscrit ainsi dans la logique de ces nouveaux instruments promotionnels (N.I.P.) dont l'essence est d'associer les objectifs de ventes immédiates de la promotion à des objectifs de fidélisation à la marque, comme au magasin ou à l'enseigne (3).

Finalement, en dépit de l'enjeu managérial du lot virtuel, les travaux portant sur le sujet demeurent encore peu nombreux. Une exploration en deux étapes a donc paru nécessaire (encadrés 3 et 4). Dans une première phase, des entretiens d'experts ont été menés pour aboutir à une meilleure compréhension des avantages et des contraintes du lot virtuel du point de vue des praticiens et expliquer ainsi son développement actuel. Dans une seconde phase, des entretiens ont été conduits auprès de consommateurs dans le but d'identifier les bénéfices et les freins perçus à l'égard du lot virtuel.

[insérer encadré 3]

[insérer encadré 4]

\section{Le lot virtuel déchiffré par les acteurs de la grande consommation}

Selon les acteurs de la distribution, le lot virtuel présente une plus grande souplesse dans la gestion du processus promotionnel, mais suppose une redistribution des coûts au sein de la chaîne commerciale. 


\section{Une plus grande souplesse dans la gestion du processus promotionnel}

De l'avis des distributeurs comme des industriels, le lot virtuel permet d'atteindre les objectifs classiquement assignés à la promotion (faire essayer, faire acheter immédiatement ou faire stocker) sans modifier pour autant les processus de gestion en place sur les plateformes de production et de logistique, comme sur le point de vente.

\section{- Souplesse dans la gestion de l'offre}

Tout d'abord, pour les industriels, le lot virtuel est facile à mettre en œuvre puisque les processus de fabrication et d'emballage restent inchangés : «Une facilité pour l'usine qui n'a pas à créer des produits spécifiques». Le lot virtuel offre par ailleurs une grande flexibilité quant aux combinaisons possibles d'offres à destination des consommateurs (unités de produits appartenant à différentes variétés ou à différentes gammes, voire à différentes marques ou différentes catégories) : «Avec le lot réel, il est impossible de faire certains lots s'ils ne sont pas ergonomiques. Avec le lot virtuel, il est possible de faire du sur mesure, c'est une sorte de lot idéal ». Pour l'industriel, le lot virtuel participe enfin du développement d'une bonne relation avec le distributeur dans le cadre d'un trade marketing efficace, car ce type d'offre promotionnelle correspond aux attentes du distributeur : "C'est un argument pour obtenir plusieurs produits sur un tract si opération promotionnelle il y a », «Pas de lot électronique = pas de place sur le prospectus s'il a pour thème le lot».

\section{- Souplesse dans la gestion des ruptures de stock}

Pour les distributeurs comme pour les industriels, le lot virtuel permet d'éviter les désagréments d'une rupture de stock liée à une mauvaise prévision des ventes de l'offre promotionnelle. Ainsi, «sur les produits spécifiques ou lots physiques, les magasins doivent commander deux mois à l'avance. S'ils ont mal prévu leurs volumes, c'est dommage, ils tombent en rupture au moment de la promotion. L'avantage du lot avec 
produit standard, c'est que ce produit est disponible sur cadencier ${ }^{l}$, donc les magasins peuvent recommander pendant la période promotionnelle s'ils n'ont pas assez de produits». Le lot virtuel se compose en effet «de produits ayant les mêmes EAN que les articles référencés en magasin, la vente sous promotion peut donc continuer au-delà de la quantité approvisionnée spécifiquement pour la promotion avec les articles présents en fond de rayon ». Le réapprovisionnement en cas de succès de l'opération promotionnelle est donc fortement simplifié.

\section{- Souplesse dans la gestion des rayons}

Le lot virtuel modifie peu la gestion de l'offre promotionnelle sur le lieu de vente. Parce qu'une opération promotionnelle assise sur des lots virtuels s'appuie sur des produits standards, la mise en rayon des produits comme le réassort par le personnel ne nécessitent pas d'ajustement dans le dimensionnement des emplacements en linéaire (hauteur des linéaires, nombre de facings...). Ce n'est pas le cas des lots réels conçus par les industriels qui ne rentrent pas toujours dans les étagères standards des distributeurs (10). De la même façon, la gestion de «l'après-promotion » se trouve également facilitée. En effet, si au terme de la période promotionnelle le lot réel implique un délotage des stocks excédentaires en vue de leur réinsertion en rayon, le lot virtuel permet une continuité sans « à-coups » dans la gestion des linéaires : «Le retour en rayon après opération promotionnelle se fait sans problème. On n'a pas le problème des lots physiques qui sont délotés ou qui envahissent les rayons s'il reste du stock sur les produits après l'opération ».

\section{Une redistribution des cô̂ts au sein de la chaîne commerciale}

Si le lot virtuel permet aux industriels de réaliser des économies sur les coûts de production et de logistique, de nouveaux coûts inhérents à la communication du lot, aux investissements informatiques ou à la tarification des produits vendus sous promotion doivent être considérés par l'ensemble des acteurs.

\footnotetext{
${ }^{1}$ Le cadencier est le document de gestion des stocks utilisé pour la gestion des linéaires en grande distribution (http://www.definitions-marketing.com/popup.php3?id_article=684).
} 


\section{- Economies sur les coûts de production et de logistique}

Pour l'industriel, une opération par lot virtuel présente de nombreuses opportunités de réduction des coûts de production et de logistique - reconditionnement, colisage spécifique, création d'un nouveau gencode - par rapport à une opération par lot réel : «Pas de coûts de confection des lots qui peuvent être très élevés en terme de carton, filmage, [...] impression éventuelle d'un pack (cartonnette), création d'un colisage différent, puis d'un gencode, gestion d'une référence supplémentaire au niveau logistique ». Outre les coûts liés à sa production même, le lot réel nécessite l'affectation de main-d'œuvre à des tâches spécifiques : «Si je dois me mettre à faire un lot physique de deux boîtes de cornetto blistées, c'est compliqué. Je dois mobiliser du personnel en usine pour dépalettiser et re-conditionner mes produits, ça me coûte cher et prend beaucoup de temps. Et puis ce sont de nouveaux codes à gérer ».

\section{- Coûts de communication}

Du côté du distributeur, le lot virtuel nécessite un budget de communication dédié : «L'inconvénient? C'est le coût de la communication, indispensable à la bonne compréhension de la mécanique par les clients», "L'opération échoue souvent par manque de communication en magasin ». En effet, parce que le lot virtuel n'existe pas physiquement, son packaging ne signale pas de lui-même l'existence d'une offre promotionnelle et il peut souffrir d'une visibilité réduite en linéaire : «Le principal inconvénient, c'est le manque d'impact en rayon et en tract: le lot virtuel n'est pas visible en linéaire par rapport aux autres lots traditionnels qui peuvent obtenir en plus d'être en "roll", c'est-à-dire dans le bas du linéaire en hyper, là ou il y a le plus de rotation... ». Moins visible que le lot réel, le lot virtuel a donc besoin de relais efficaces en termes de publicité sur le lieu de vente pour matérialiser l'avantage promotionnel qui s'y trouve associé.

\section{- Coûts des investissements informatiques}

Le lot virtuel suppose également l'installation de caisses « intelligentes », et partant, des investissements matériels conséquents pour les distributeurs : «Techniquement, le lot virtuel n'est pas évident à mettre en place, car il suppose que toutes les caisses de l'ensemble des magasins de l'enseigne soient paramétrées pour que la réduction se fasse directement en caisse. Certaines enseignes indépendantes ne peuvent donc toujours pas 
faire de lot virtuel ». On observe ainsi un équipement progressif mais en ordre dispersé des différentes enseignes françaises. Si Carrefour a été pionnier, Intermarché n'a suivi que très tardivement et «Système U reste encore en grande partie en dehors du jeu ».

\section{- Tarification différenciée difficile à évaluer}

Le lot virtuel permet enfin à l'industriel de mieux contrôler son budget promotionnel. En effet, dans le cas du lot réel, l'industriel propose sa marchandise au distributeur à un prix temporairement réduit. La durée de l'offre promotionnelle est restreinte et ne présente généralement pas de limite en termes de quantités achetées : le distributeur a donc l'opportunité d'acheter à un prix avantageux un plus grand nombre d'unités que celles qu'il va réellement vendre pendant la période promotionnelle. En revanche, avec le lot virtuel, seules les unités promotionnelles effectivement passées en caisse pendant la durée de l'opération bénéficient du tarif promotionnel. Le distributeur passe ainsi d'une gestion «forfaitaire » de la promotion à une gestion « au rendement » beaucoup plus efficace sur le plan budgétaire. Néanmoins, l'un des experts interrogés a souligné qu'il était parfois difficile d'identifier le véritable nombre d'unités écoulées dans le cas d'un lot virtuel : «Les fabricants ont peur d'une dérive ». Par ailleurs, il semble que le prix refacturé par les distributeurs aux industriels pour les produits écoulés gratuitement puisse être le prix de vente consommateur dans le cas du lot virtuel, et non le prix de revient comme dans le cas du lot réel : «Dans l'exemple de structures plus petites, le prix auquel est refacturé le lot virtuel par le distributeur peut être le prix de vente consommateur [...] alors que dans le cas d'un produit gratuit donné par le fabricant, il coûte à ce dernier le coût de revient ». Or, le prix de vente consommateur est supérieur au prix de revient puisqu'il intègre les marges arrière du distributeur. Les unités auxquelles l'industriel accorde une réduction seraient donc effectivement les unités vendues au consommateur lors de l'opération, mais elles pourraient lui être facturées à un prix plus élevé par le distributeur.

Globalement, le lot virtuel permet donc d'atteindre les objectifs classiquement assignés aux offres promotionnelles, tout en favorisant des économies tout au long de la chaîne commerciale. Il apparaît ainsi comme une technique promotionnelle intéressante pour les acteurs de la grande consommation et semble appelé à poursuivre un développement 
rapide dans la distribution des biens de consommation courante. Maintenant, avant de proposer des recommandations pertinentes pour optimiser sa mise en œuvre, il reste toutefois à comprendre comment les consommateurs le perçoivent relativement au lot réel.

\section{Le lot virtuel tel que perçu par les consommateurs}

D'un point de vue économique, l'analyse des entretiens révèle que les consommateurs semblent considérer le lot réel et le lot virtuel comme deux techniques promotionnelles similaires: "J'ai l'impression que c'est un peu pareil. Tu as trois produits et on t'explique bien qu'il y en a un troisième gratuit. La technique me paraît la même ». Néanmoins, la comparaison des perceptions par les consommateurs du lot virtuel par rapport au lot réel met en lumière un certain nombre de bénéfices et de freins spécifiques au lot virtuel.

\section{Les bénéfices associés au lot virtuel}

Les consommateurs associent deux types de bénéfices au lot virtuel comparativement au lot réel : un bénéfice hédonique et un bénéfice écologique.

\section{- Bénéfice hédonique}

Au regard du discours des consommateurs, la promotion de lots composés de produits hétérogènes est plus valorisée dans le cadre du lot virtuel que du lot réel. En effet, le lot virtuel reposant sur des produits hétérogènes offre aux consommateurs la liberté du choix des parfums qu'ils aiment ou qu'ils souhaitent découvrir : «Je préfère choisir moi-même. On peut avoir l'opportunité de tester quelque chose de nouveau ». Ce choix est en revanche contraint dans le cadre du lot réel. La présence d'un parfum non apprécié dans un lot réel hétérogène devient dès lors un frein important à son achat : «Si dans le lot il y a un parfum que je n'aime pas, je ne le prends pas ». 


\section{- Bénéfice écologique}

Contrairement au lot réel, un bénéfice écologique associé au lot virtuel s'est dégagé du discours des consommateurs. Mus par une sensibilité accrue aux conséquences environnementales de leurs choix, les consommateurs ont tendance à condamner l'usage abusif de sur-emballages. La réduction conséquente des sur-emballages, inhérente au lot virtuel comparativement au lot réel, conduit ainsi certains consommateurs à privilégier le lot virtuel : «Personnellement, je suis contre le fait que les lots soient préparés et emballés, surtout par rapport à une notion de sur-emballage. Ce n'est pas très écologique ».

\section{Les freins associés au lot virtuel}

Si le lot virtuel présente des bénéfices d'ordre hédonique et écologique par rapport au lot réel, de nombreux freins à l'achat du lot virtuel sont cependant évoqués par les consommateurs. Ces freins associés au lot virtuel sont au nombre de trois : sa moindre visibilité, sa plus grande complexité et l'incertitude quant à l'obtention de son bénéfice monétaire.

\section{- Moindre visibilité}

Le lot virtuel est perçu comme moins visible que le lot réel : «Le lot qui est attaché est plus visible que l'autre (le lot virtuel) ». Le lot virtuel est en effet annoncé par le distributeur sur prospectus et par affichage en rayon dans le magasin. Mais, contrairement au lot réel, il n'est pas présenté sur le packaging du produit ce qui rend la promotion moins facile à détecter pour les consommateurs : "L'affichage met bien en avant l'idée de lot, mais en terme de packaging, on est en dehors de la norme qui est de regrouper le produit». Ainsi, le lot virtuel est moins facilement perçu en magasin. Conséquence de cette moindre visibilité du lot virtuel, les consommateurs craignent de passer à côté de la promotion : "Les gens ne vont en prendre que deux, ils ne vont pas faire attention au fait qu'ils pourraient avoir le troisième gratuit ». L'attitude à l'égard du lot virtuel s'en trouve dès lors affectée.

\section{- Effort cognitif plus important}


Le lot virtuel est perçu comme moins clair que le lot réel. Il amène les consommateurs à une réflexion plus intense en ce qui concerne la décision d'achat alors que le lot réel traduit immédiatement un «signal promotionnel » : «Il faudrait que je fasse... un petit chemin intellectuel pour me dire que si je prends deux bouteilles, j'en ai une gratuite. Alors que dans le cas précédent, les deux sont associées, le visuel me donne tout de suite l'information que le produit est en promotion ». En raison de ce manque de clarté du lot virtuel, les consommateurs craignent de se tromper quant au nombre de produits à prendre pour bénéficier de l'avantage: «Quand ce n'est pas emballé, c'est un peu ennuyeux car on doit toujours compter combien on en a pris... on peut se tromper ». La communication du lot réel est moins complexe que celle du lot virtuel. Le premier présente le pack et son prix alors que le second doit communiquer simultanément l'offre de base (produit et prix à l'unité) et le contrat correspondant à l'offre virtuelle (conditions et prix du lot). Dans l'exemple du lot réel homogène Tahiti (encadré 4), deux prix sont affichés : le prix du lot $(5 €)$ et le prix au litre $(5,56 €)$. Pour un lot homogène mais virtuel (lot Brioches Champion), trois prix sont indiqués : le prix du lot $(3 €)$, le prix à l'unité $(1,50 €)$ et le prix au kilo $(3,50 €)$.

\section{- Incertitude quant à l'obtention du bénéfice économique en caisse}

Le lot virtuel est associé à une plus forte incertitude par les consommateurs. En effet, certains consommateurs émettent des doutes sur la prise en compte effective de l'avantage promotionnel attaché au lot virtuel lors du passage en caisse alors que cet avantage est considéré comme certain pour le lot réel : "Quand il y a un lot bien groupé, il n'y a pas de problème. Le lot passe avec un seul code». Les consommateurs doivent donc redoubler de vigilance lors de leur passage en caisse : "Il faut que je fasse attention à mon ticket de caisse à la sortie (...) il faut que je vérifie que la promotion a bien été décomptée ». Toutefois, cette incertitude est atténuée par le type d'enseigne considéré. Pour certains consommateurs, les «grands groupes» comme Auchan ou Carrefour sont perçus comme disposant d'un équipement informatique à la pointe de la technologie. Ces enseignes sont donc moins risquées du point de vue du consommateur car elles prennent automatiquement en compte tous types de promotions. Le consommateur est sûr que le lot virtuel sera bien décompté lors de son passage en caisse : "Chez Auchan, ce sont des caisses sophistiquées. C'est calculé 
automatiquement... il y a un travail automatique, informatisé qui fait que lorsque l'on en passe deux pour le même client la troisième ne sera pas comptée, quel que soit l'ordre de présentation des produits. En grande surface, je ne crains rien ».

\section{Conclusion}

Les entretiens d'experts ont globalement souligné les nombreux avantages du lot virtuel, qui offre l'opportunité de réduire les coûts de chacun des partenaires tout en les incitant à faire des économies conjointes. En particulier, le lot virtuel permet d'éviter les coûts industriels de constitution et de stockage du lot. Deux sources de coûts spécifiques au lot virtuel ont néanmoins retenu l'attention des professionnels : les investissements informatiques imposés au distributeur et l'éventuelle refacturation à l'industriel du lot au prix de vente consommateur. Si les premiers s'amortissent avec le temps, les suivants devraient diminuer dans le cadre de la mise en application des dispositions de la loi Dutreil II. Cette loi, entrée en vigueur le $1^{\text {er }}$ janvier 2006, impose en effet la réintégration progressive des marges arrière dans le seuil de revente à perte (pour l'heure, la part excédant $15 \%$ du prix unitaire facturé), ce qui devrait à terme réduire l'écart entre prix de vente au consommateur et prix de revient. Malgré l'émergence de coûts spécifiques inhérents au lot virtuel, celui-ci présente suffisamment d'avantages pour laisser envisager la poursuite de son essor dans la grande distribution.

Toutefois, du fait même de son faible degré de matérialisation, le lot virtuel peut susciter des perceptions négatives de la part des consommateurs. S'il présente des bénéfices hédonique et écologique pour les consommateurs, le lot virtuel génère des coûts cognitifs supplémentaires dans le processus de décision, coûts en particulier liés à l'incertitude qui pèse sur l'obtention effective du bénéfice dans le cadre du lot virtuel. Ces coûts pourraient en effet modérer l'intérêt global porté au lot virtuel. Afin de réduire les freins associés au lot virtuel, nous formulons des recommandations managériales. 


\section{Favoriser les pratiques collaboratives entre industriels et distributeurs}

La littérature académique a mis l'accent ces dernières années sur la nécessité pour les membres d'un canal de distribution d'agir de manière coordonnée pour améliorer l'efficacité $(4,14)$. Il s'agit pour les différents acteurs de se concerter étroitement et durablement pour définir quand et comment exécuter les activités créatrices de valeur. La gestion des promotions et des animations sur le point de vente constitue l'un des aspects de ces pratiques de collaboration. Les entretiens d'experts ont en particulier révélé l'importance de la communication en magasin qui constitue la seule «matérialisation » de la promotion. Cette dernière est d'autant plus importante qu'elle assure la visibilité et le succès d'une opération assise sur la technique du lot virtuel. En effet, les consommateurs ont souvent déploré le manque de visibilité des lots virtuels qui constitue un frein certain à son utilisation. Afin d'accroître la visibilité du lot virtuel en magasin, il paraît plus opportun d'organiser des animations hebdomadaires assises sur la multiplication des promotions par lot virtuel sur l'ensemble du magasin, plutôt que de mener ces opérations au coup par coup. Ces animations seraient l'occasion d'habiller le point de vente d'une signalétique uniforme plus lisible, voire d'installer des îlots spécifiquement dédiés à la promotion par lot virtuel à certains emplacements stratégiques.

\section{Promouvoir la transparence dans les relations entre industriels et distributeurs}

L'un des présupposés de ces pratiques collaboratives est l'instauration d'un degré de confiance élevé entre les fabricants et les distributeurs. Or, les entretiens d'experts ont montré qu'à l'heure actuelle, le nombre de lots réellement écoulés ainsi que leur prix de refacturation manquent de transparence. Des négociations demeurent donc nécessaires entre industriels et distributeurs afin de fixer plus clairement les termes du contrat associé à la technique du lot virtuel.

\section{Familiariser les consommateurs au lot virtuel}

Pour les consommateurs, le lot virtuel demeure une technique promotionnelle difficile à comprendre. Afin d'aider les consommateurs dans leur apprentissage de cette technique 
promotionnelle, l'usage du prospectus paraît opportun. Le prospectus du magasin peut en effet offrir un format souple et peu contraignant pour expliquer la nature du lot virtuel. Il s'entoure dès lors d'une fonction pédagogique à destination des consommateurs. Ainsi, il est important d'insister sur l'explication des conditions à respecter pour pouvoir profiter de l'avantage, mais également sur la nature automatisée de la validation du respect de ces conditions lors du passage en caisse.

\section{Communiquer sur les bénéfices écologiques associés au lot virtuel}

D'un point de vue plus prospectif, le lot virtuel pourrait également bénéficier de la sensibilité accrue des consommateurs aux conséquences environnementales de leurs choix (12), comme du développement d'un droit communautaire spécifique (directive 94/62/CE du 20 décembre 1994 relative aux emballages et à leurs déchets (11)). Du point de vue des distributeurs, une communication environnementale portant sur les bénéfices écologiques liés à la réduction des sur-emballages inutiles peut favoriser le développement du lot virtuel tout en développant la légitimité de l'enseigne auprès des consommateurs (7).

En conclusion, le lot virtuel, par ses caractéristiques, devrait poursuivre son essor au sein des techniques promotionnelles plébiscitées par les acteurs de la grande consommation. Introduit par les grands groupes (Carrefour, Auchan...), le lot virtuel se développera in fine dans les petites enseignes et enseignes indépendantes bénéficiant dès lors d'une familiarité accrue du consommateur à cette technique promotionnelle.

Si cette recherche à vocation exploratoire met en évidence les bénéfices et les freins associés au lot virtuel, elle pourra être complétée par des recherches de nature plus quantitative. De telles recherches permettraient de mesurer les comportements effectifs des consommateurs en magasin face à ce nouvel instrument promotionnel et d'en évaluer les coûts et les bénéfices. Plus particulièrement, de futures recherches pourraient s'attacher à évaluer l'influence de la complexité cognitive associée au lot virtuel sur la perception de l'obtention de l'avantage économique. 


\section{Références}

(1) Bardin L. (2003), L'analyse de contenu, Le psychologue, Paris, PUF.

(2) Chandrashekaran R. (2004), The influence of redundant comparison prices and other price presentation formats on consumers' evaluations and purchase intentions, Journal of Retailing, 80, janvier, 53-66.

(3) Debos F. (2006), N.I.P (Nouveaux Instruments Promotionnels) et promotions des marques : vers une synergie nécessaire, in La communication persuasive 2, D. Benoît, A. Mucchielli et Y. Chirouze (eds.), Paris, Eska, 6-16.

(4) des Garets V. (2000), Les relations distributeur-fabricant et les modalités de leur coordination, in N. Fabbe-Costes, J. Colin et G. Paché (eds), Faire de la recherche en logistique et distribution ?, Paris, Vuibert-Fnege, 109-126.

(5) Desmet P. (1999), Le lot virtuel: une application d'un marketing interactif de masse, Décisions Marketing, 16, janvier-avril, 61-66.

(6) Drèze X. et Bell D. (2003), Creating win-win trade promotions: theory and empirical analysis of scan-back trade deals, Marketing Science, 22, hiver, 16-39.

(7) Gauthier C. et Renaud E. (2005), L'impact de la communication environnementale. Le cas E. Leclerc, Décisions Marketing, 39, juillet-septembre, 21-32.

(8) Guiltinan J.P. (1987), The price bundling of services: a normative framework, Journal of Marketing, 51, avril, 74-85.

(9) Michalowska A. (2002), Distributeurs : quelles attentes envers leurs fournisseurs ?, Marketing Magazine, 66, janvier, 16.

(10) Ong B.S., Ho F.N. and Tripp C. (1997), Consumer perceptions of bonus packs: an exploratory analysis, Journal of Consumer Marketing, 14, avril, 102-112.

(11) Pinet B. (2004), Développement durable et marketing de l'emballage, Revue Française du Marketing, 200, décembre, 27-38.

(12) Schwepker C.H. Jr. et Cornwell B. (1991), An examination of ecologically concerned consumers and their intention to purchase ecologically packaged products, Journal of Public Policy \& Marketing, 10, automne, 77-101.

(13) Stremersch S. et Tellis G. (2002), Strategic bundling of products and prices: a new synthesis for marketing, Journal of Marketing, 66, janvier, 55-72. 
(14) Tyndall G., Gopal C., Partsch W. et Kamauff J. (1998), Ten strategies to enhance supplier management, National Productivity Review: The Journal of Organizational Excellence, 17, été, 31-44. 


\section{Encadré 1 : Une typologie des lots virtuels}

Les lots virtuels se distinguent en fonction des conditions d'obtention de l'avantage proposé (type et nombre de produits à acheter) :

- achat simultané de plusieurs articles identiques (par exemple des paquets de biscuits strictement identiques) : on parle alors de lot «jumelé » ou lot « homogène »;

- achat simultané de plusieurs articles du même type mais de variétés différentes (par exemple différents parfums de glaces) : on parle alors de lot « variété » ou lot « hétérogène »;

- achat simultané de plusieurs articles différents appartenant à la même gamme de produits d'une marque (par exemple un camembert Président et une plaquette de beurre de cuisson Président) : on parle alors de lot « gamme » ou lot « hétérogène ».

Dans chacun de ces cas, l'avantage offert diffère selon trois dimensions :

- sa nature : produit gratuit versus réduction monétaire exprimée en euros ou en pourcentage ;

- son ampleur : les formes les plus courantes sont «pour un acheté, le deuxième à moitié prix » (Buy One Get One Half Price), «pour deux achetés, le troisième gratuit »(Buy Two Get One Free), «pour un acheté, le deuxième gratuit »(Buy One Get One Free);

- sa temporalité : obtention immédiate (réduction immédiate en caisse ou produit gratuit) versus différée (réduction monétaire portée sur le compte de la carte de fidélité du magasin).

Source : Philippe Ingold (http://www.cles-promo.com/techniques/techniques.htm) 


\section{Encadré 2 : Développement du lot virtuel en France}

Alors qu'il existe aux Etats-Unis depuis les années 80, le lot virtuel ne prend son essor en France que très tardivement, en 1994, date à laquelle la société Catalina implante ses premiers médias de caisse. L'utilisation du lot virtuel demeure relativement limitée en France jusqu'au déploiement, quelques années plus tard, de technologies de caisses «intelligentes » permettant de rendre immédiat l'avantage promotionnel qui lui est associé. Dès lors, le lot virtuel devient une technique d'animation incontournable pour certaines enseignes, comme en témoignent les prospectus entiers qui lui sont désormais consacrés («Les gratuits » chez Auchan du 5 au 11 octobre 2005, «Promo Banco » chez Casino du 22 mars au 1er avril 2006). Une pige des prospectus du magasin Auchan de La Chapelle Saint-Aubin (72) réalisée au sein de la société MarketingScan montre d'ailleurs que le nombre de lots virtuels communiqués sur prospectus y a été multiplié par dix entre 2003 et 2005 (60 au dernier trimestre de 2003 contre 543 au dernier trimestre de 2005).

En 2006, le lot virtuel représente en France 22\% des offres promotionnelles communiquées sur prospectus selon les chiffres transmis par l'entreprise Le Site Marketing. 82\% des lots virtuels sont des offres immédiates. Par ailleurs, la ventilation des lots virtuels en fonction de l'ampleur de l'avantage promotionnel est la suivante : «pour un acheté, le deuxième à moitié prix »(44\%), «pour deux achetés, le troisième gratuit » $(25 \%)$, «pour un acheté, le deuxième gratuit » $(9 \%)$. 


\section{Encadré 3 : Méthodologie des études menées auprès d'experts et de consommateurs}

Première étape : six entretiens semi-directifs individuels ont été conduits auprès de différents experts de la commercialisation de biens de consommation courante (Colgate-Palmolive, Unilever, Yoplait), de la distribution (Monoprix) et de l'étude des promotions (Le Site Marketing) et du comportement réel d'achat des consommateurs (Catalina). Ces entretiens d'une quarantaine de minutes en moyenne ont été menés en face à face ou par téléphone. Ils ont principalement abordé deux thèmes :

- l'utilisation du lot virtuel, dans ses dimensions stratégique et historique ;

- l'identification des bénéfices et des contraintes caractéristiques du lot virtuel relativement au lot réel.

Deuxième étape : des entretiens semi-directifs individuels d'une durée moyenne de 30 minutes ont été menés auprès de 18 consommateurs âgés de 26 à 59 ans. Les thèmes abordés font référence à la compréhension de l'offre promotionnelle présentée, à l'attitude à son égard et enfin aux motivations et freins perçus à l'idée de bénéficier de cette offre promotionnelle. Les stimuli promotionnels étaient au nombre de quatre (encadré 4 pour un exemple), soumis successivement aux interviewés dans un ordre de présentation variant d'un entretien à l'autre.

Pour choisir les stimuli promotionnels, un corpus d'offres promotionnelles réelles a d'abord été créé à partir d'un travail d'observation en magasin et d'analyse de prospectus distribués durant le mois de novembre 2005. A l'issue de cette phase d'observation, plusieurs catégories de produits faisant souvent l'objet de promotions sous forme de lots réels et virtuels ont été sélectionnées : les shampooings, les gels douche, les boissons gazeuses, etc. Le nombre de stimuli promotionnels finalement retenu a été limité à quatre afin d'éviter tout effet de lassitude chez l'interviewé lors de la conduite de l'entretien. Ce nombre de quatre offres promotionnelles permet de croiser aussi bien le caractère réel versus virtuel du lot, que la nature homogène versus hétérogène des produits assemblés dans le lot. La valeur relative de l'avantage promotionnel pour chacune de ces quatre offres est identique $\left(2=3^{\text {ème }}\right.$ gratuit $)$.

Les entretiens ont fait l'objet d'une analyse de contenu verticale et horizontale favorisant l'identification de la régularité d'occurrence des thèmes (1). 


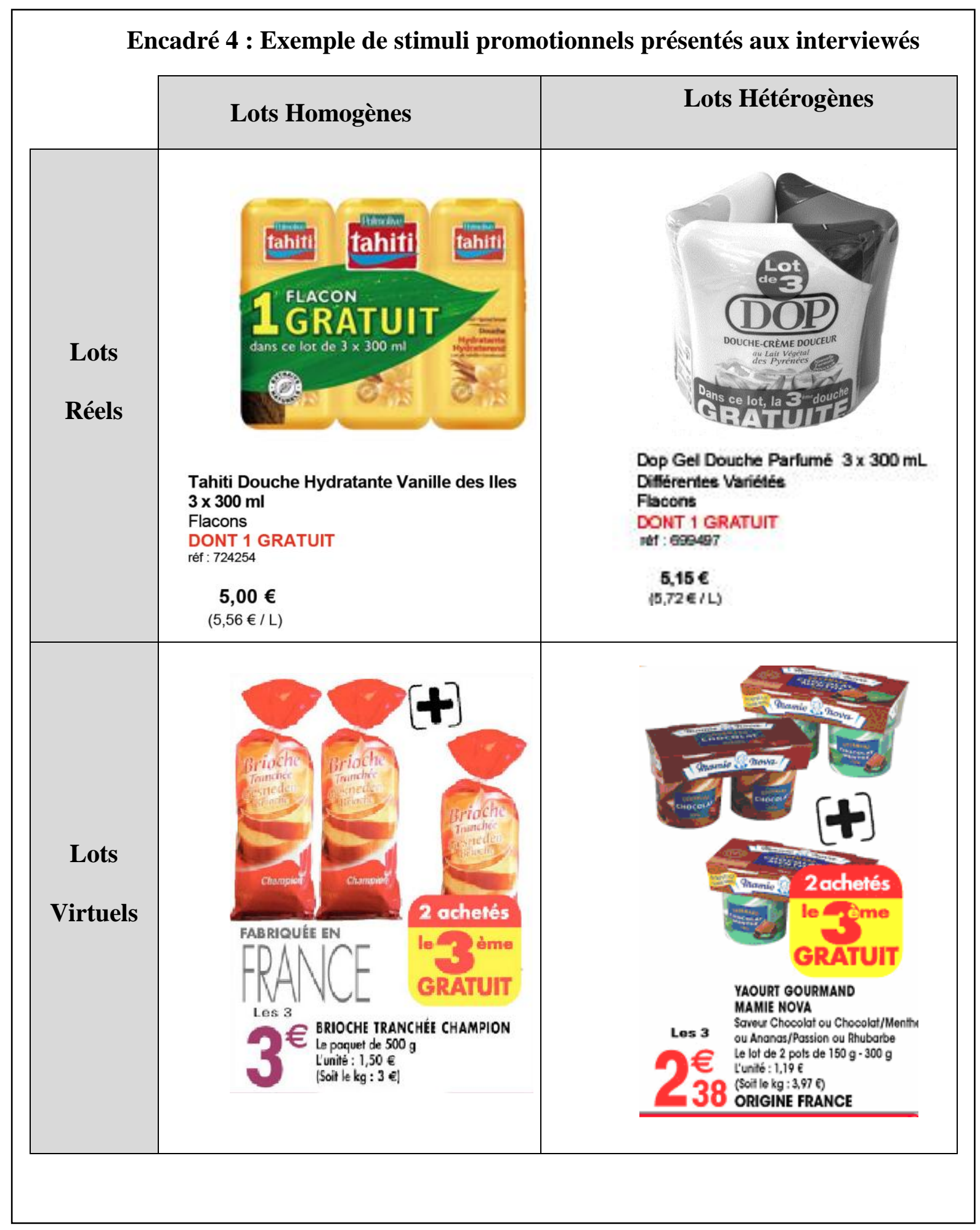

